\title{
A Multimedia-based City Information System
}

\author{
Kranthi Kumar.Ravi. \\ P.J.Narayanan \\ C.V.Jawahar \\ kranthi@gdit.iiit.net \\ pjn@iiit.net \\ jawahar@iiit.net \\ Indian Institute Of Information Technology, \\ Hyderabad, INDIA
}

\begin{abstract}
Plenty of information is available today from different sources about a particular geographical area. However, none of it is reliable when different needs arise like for tourists, city planners, administrators, and lay people. We have designed a multimedia based city information system which attempts to solve this problem. In this paper we explain the design and implementation of our system. The paper discusses in detail the need for such a system, how it compares with similar existing systems and the features expected in such a system. It also discusses our implementation of the system and the future work we want to pursue in this direction.
\end{abstract}

\section{INTRODUCTION}

Information is available in plenty today from different sources about many aspects of a fast-changing, geographic, entity such as a modern city. Many pieces of this information could be of great use to some section of the citizens and administrators. Different governmental/non-governmental departments and sections of the society hold different pieces of information. They are rarely brought together into a single forum to the benefit of those who need them. Even if they are, there are few avenues to present them to a wide cross section of users in an economical and user-friendly manner. A city presents adequate challenges and sufficient diversity at an appropriate scale for this problem. Hence we concentrated on a city for the initial phases of the development of our system.

We have developed the Multimedia-based City Information System (MmCIS) for a city, taking Hyderabad as the case study. The system provides a variety of information about the city, its history, and its citizen services through the friendly medium of interactive multimedia. Information accuracy is very important in systems like this, when various aspects of the city change rapidly. The system is meant for professionals as well as for minimally IT-savvy lay users. Multimedia, with its human-oriented interfaces such as text, pictures, video, and sound, is the ideal medium of delivery to such a cross section of users. The actual user categories and the information delivery is quite diverse Depending on the end-user.

The MmCIS system is geographical in essence as the geography forms the basis for any information system about a city. Some geographical information is available about a typical city today, such as maps, road networks etc. However, the geographical information available about most cities -- electronically or otherwise -- is old and out-dated. It can be safely said about any Indian city that even the best map available about it cannot be trusted to plan a route from one point to another within the city. We, therefore, aim to update and validate the available geographic information periodically using more complete sources such as satellite images[1][6], direct human knowledge, etc. In fact, the side- 
product of our project that could be of most wide appeal to the people may be the map of the city itself. Other information, such as tourist advisories, bus-route planner, etc., will be overlaid on top of the basic geography.

We present the goals of the system and how it compares with similar systems in the next section. Section 3 discusses how these goals are realized using the different modes of information available today. Section 4 shows the design of our system and the current state of its development. Section 5 presents avenues for future work.

\section{SYSTEM}

\subsection{System Goals and Design}

The MmCIS has multi-fold goals. The whole information as we envisage can be categorized into different complementary sub-knowledge divisions representable in the form of layers. Each category of information, as we explain further, can be separated from the other with the only common characteristic being the geographical layer of the map. For example, a road-network-layer can be modeled and updated independent of another layer which gives the environmental composition of the city. Each of the layers would complement the information provided by other layers.

Depending on the target consumers of the system, different layers of the system would be combined for the display to provide most useful and accurate information for the specific category of the user. MmCIS also provides specific information about the economic conditions of different localities in the city. This would help the advertising people and sales persons to go for targeted-advertising and mass promotion programs for their products. This information would be dynamically updated and would represent near-accurate information about the actual standard of living of the people living in each area.

The MmCIS would also be useful to administrators to check the growth of the city. For example, administrators can check for any new buildings coming up in a particular place and for which permission was not specifically taken from the government - and could accordingly levy taxes or stop the unauthorized construction activities. This information can be gathered fom high-resolution satellite images and by subjecting them to various digital image processes. Administrators can also plan for new roads to be laid-out or roads to be expanded to account for the increasing traffic density in certain areas.

The MmCIS could also adapt to the particular user. For example, if the user of the system is constantly looking for information related to city road network layer, the system would concentrate more on the road network layer and analysis corresponding to that layer. Similarly, if another user is often using the environment layer of the system, the system would highlight environment layer and tries to analyze the specific information which would complement the use of the particular component.

The potential users of the system can be categorized into:

- Casual city users.

- Tourists.

- City Planners.

- $\quad$ Advertising and Sales Agencies. 
- Administrators.

- $\quad$ Other service providers.

This is not an exhaustive list of the users, but only some of the possible users of the system as we see at this point of development. The system could be composed of several layers, some of which are:

- $\quad$ Core city Layer.

- Map Layer.

- $\quad$ City Road Network Layer.

- Transportation Layer.

- Railway Services Layer.

- Historic Places Layer.

- Satellite Image Layer.

- Environment Layer.

- General Information Layer.

We explain the different layer composition, the source of data for each of the layer, who would be the major benefactories of each layer in the table give below.

\begin{tabular}{|c|c|c|c|c|}
\hline Layer Name & $\begin{array}{l}\text { What the Layer } \\
\text { contains }\end{array}$ & Representation & Data Sources & Target Users \\
\hline Core City & $\begin{array}{l}\text { Outline of the whole } \\
\text { city, main roads. }\end{array}$ & $\begin{array}{l}\text { Text overlaid over } \\
\text { map }\end{array}$ & $\begin{array}{l}\text { Cartographic } \\
\text { information, images } \\
\text { from satellites. }\end{array}$ & $\begin{array}{l}\text { Casual city users, } \\
\text { Administrators }\end{array}$ \\
\hline $\begin{array}{l}\text { City Road } \\
\text { Network }\end{array}$ & $\begin{array}{l}\text { Inter-connections of the } \\
\text { roads in the city }\end{array}$ & Images. & $\begin{array}{l}\text { Satellite images [3], } \\
\text { administrators, } \\
\text { humans. }\end{array}$ & $\begin{array}{l}\text { Casual city users, } \\
\text { Administrators, City } \\
\text { planners }\end{array}$ \\
\hline Transportation & $\begin{array}{l}\text { Transporatation } \\
\text { facilities available in } \\
\text { the city }\end{array}$ & & $\begin{array}{l}\text { Manuals published by } \\
\text { transport corporation, } \\
\text { humans. }\end{array}$ & $\begin{array}{l}\text { Tourists, Other } \\
\text { service providers. }\end{array}$ \\
\hline Historic Places & $\begin{array}{l}\text { Details of historic } \\
\text { places, how to reach } \\
\text { them, best times to visit } \\
\text { etc. }\end{array}$ & $\begin{array}{l}\text { Video }+ \text { map } \\
\text { explaining historic } \\
\text { relevance of the } \\
\text { location. }\end{array}$ & $\begin{array}{l}\text { Tourism department, } \\
\text { tourist guides and } \\
\text { others who know the } \\
\text { places. }\end{array}$ & $\begin{array}{l}\text { Tourists, Other } \\
\text { serivice providers. }\end{array}$ \\
\hline $\begin{array}{l}\text { Railway } \\
\text { Services }\end{array}$ & $\begin{array}{l}\text { Trains arrival and } \\
\text { depature, special } \\
\text { services for tourists }\end{array}$ & $\begin{array}{l}\text { Text, image maps, } \\
\text { video }\end{array}$ & $\begin{array}{l}\text { Manuals by Indian } \\
\text { Railways, other people. }\end{array}$ & Tourists \\
\hline
\end{tabular}

Table 2.1: MmCIS Layer Composition, Data Sources and Major Benefactors. 


\subsection{Other Similar Systems}

There are other systems which more or less are based with a similar fundamental idea in mind. One major classification of them is the GIS and the other major one are web-based systems like expedia.com.

In the strictest sense, a "GIS is a computer system capable of assembling, storing, manipulating, and displaying geographically referenced information, i.e. data identified according to their locations"[7]. Geographical Information Systems (GIS) are used widely for similar purposes today, especially by town planners and administrators. The MmCIS system, while being similar to a typical GIS system due to its geographical character, has somewhat different goals. Firstly, our system is aimed at thousands of users from different cross sections. Information may be retrieved from a $\mathrm{CD}$ on a home computer or accessed from a Web-page over different access mechanisms. Real-time information may be needed on-line from a moving vehicle using either of these media. Traditional GIS systems tend to be prohibitively expensive for such a wide circulation and hence are not suitable as the backbone of a system like MmCIS. Equally importantly, we see different modes of information being delivered to the user as per his or her preference. GIS systems are not suitable for these applications as they concentrate more on the representation and analysis of geographyreferenced data stored in a database.

The second type of systems which are closer to our system are web-based free services like expedia.com[8]. These web-based systems while differing from the GIS in that there is not much analysis of a geography-referenced data. These systems are targeted at tourists. The services offered by a typical system like this are route paths from one place to another, reservation facilities in hotels, air-travel timings and reservations etc to name a few. Our system offers far many facilities and serves a wide audience than these kinds of systems.

\subsection{Adapting to the requirements of a particular category of users}

The MmCIS keeps track of all the instances of the use of the system by a particular user. These logs inturn are processed and details of the sections which are being frequently being used by a particular user are extracted out. This will help out in the system adapting to the requirements of a particular user. A very scaled down example in simple terms is: , if a user frequently uses route information, especially say from location $\mathrm{x}$ to location $\mathrm{z}$, the system will actually compute this information whenever the user starts the system even before the user asks for the information the next time. Also, if the user hardly uses information from say roads layer, the system would not load any information related to the road layer into the memory, but would use the same for something which the user accesses frequently.

\section{REALIZATION OF THE SYSTEM:}

How the above goals can be realized using the information tools already available is already explained in table 2.1.

We plan to deliver the information using dedicated servers which would be updated from time-to-time as and when new information is available. This holds with all online, wired and wireless delivery systems - on the web, WAP enabled devices, telephone, etc. We plan to use standard tools and mechanisms to deliver information over offline media like CD-ROMs; and online ones like web and WAP devices. Special tools to convert text to speech in English and Indian languages may need to be developed to deliver information over a telephone. 


\section{SYSTEM IMPLEMENTATION}

\subsection{Language and Platform}

The whole system is sub-divided into several layers. Each layer draws its information from various sources as was detailed in the previous section. Our present implementation of the MmCIS includes the core city, map, road network, railway services, historic places and general information layers enhanced with multimedia information. We have implemented the system on MS Windows platform using visual $\mathrm{C}++$, directx and some sub-components using Macromedia shockwave and flash.

To generate the core city layer, we have used available cartographic information. In our implementation, the city layer shows the basic outline of the city, main roads, etc. It is represented as text information overlaid over a map as shown in Figures 1 and 2. The map is digitized instead of storing the whole information - discarding unnecessary data. For the road network layer, we have used information from humans along with cartographic information.

For the railway services layer, we have used maps available from Indian Railways and have super-imposed them over the core city layer after sufficient scaling. The railway services layer we have implemented also includes audio information about timings of arrival and departure of train services. The information included by us is augmented with different points in the city from which one can make railway reservations. We have obtained this information from the World Wide Web.

\subsection{Other Features}

A prominent sub-component available in the system gives the shortest path between various places. The component bases it's conclusions from different layers, and other heurists about the traffic density etc. We soon plan to support these heuristics with actual data collected from city administrators and by processing satellite images[1][2][6]. It takes into account the type of roads like national highways, one-ways, roads under repair etc[3].

Current implementation of the system is portable to stand-alone versions on CD-ROMs, DVD-ROMs and the Internet. The system provides output to the user using the most advanced facilities available as of now to give the user most natural experience with out subjecting him to go for 'search-and-learn'. It is more than just a catalogue of information. The system is interactive and provides lots of video and audio information which is informative than a textual or map information.

For example, a user may just click on the start location and then the end location and the system provides him detailed information about the best possible path to take, along with intricate details in the form of audio. While at the same time, the system would also indicate to the user any historic places of importance along the path being taken. By clicking on any of these places, a user can view videos of the places explaining their historic relevance. (See Figure 2) The user at the same time would be provided with only a brief overview and is prompted whether he/she wants to know further details about the monument/place and would accordingly be served with the details. 


\section{FUTURE WORK}

As of now, the system implements the layers mentioned in the previous section and needs to be integrated with GPS, etc. We also plan to make it compatible with WAP enabled devices so that information dissemination would be lot faster, easier and convenient. We also plan to work on the satellite image layer. This would form the most important part of our system. The layer helps us correct any errors in other layers using accurate information available from processing the high-resolution satellite images. The issues which arise include processing in the compressed domain, etc. The incorporation of this layer makes the system most accurate; and also when augmented with GPS.

While information can always be updated to make the system most reliable, another issue which we would like to tackle is changing the user-interface. We already know that the user-interface constitutes the most important part of the whole system. We would like to make it a pluggable module; so that we would be able to incorporate any new developments coming in this area to make the user-interface most natural and easier.

\section{REFERENCES}

[1]. Chen.P.H. and Wintz,P.A.[1976] Data Compression for Satellite Images, TR-EE-76-9, School of Electrical Engineering, Purdue University.

[2]. Gregory.A.Baxes, Digital Image Processing - Principles \& Applications, $\quad$ John Wiley \& Sons, NewYork. [3]. Huertas,A., Cole.W., and Nevetia, R.[1990] Detecting Runways in Complex Airport Scenes, Computer Vision, Graphics, Image Proc., Vol51, no.2, pp 107-145.

[4]. Pavlidis, T. [1972] Segmentation of Pictures and Maps through Functional Approximation, Comp. Graph., Image

Proc., Vol 1, pp. 360-372.

[5]. Rafael.C.Gonzalez, Richard.E.Woods, Digital Image Processing, Addison-Wesley, New York.

[6]. Schonengertt, R.A. [1983], Techniques for Image Processing and Classification in Remote Sensing, Academic Press, New York.

[7] http://www.usgs.gov/research/gis/title.html U.S. Department of the Interior, U.S. Geological Survey, Reston, VA, USA

[8] http://www.expedia.com

\section{IMAGES:}

1. MmCIS showing shortest path between two locations.

2. MmCIS showing the menu for one of the places of historical importance.

3. MmCIS showing video information of a historically prominent place. 


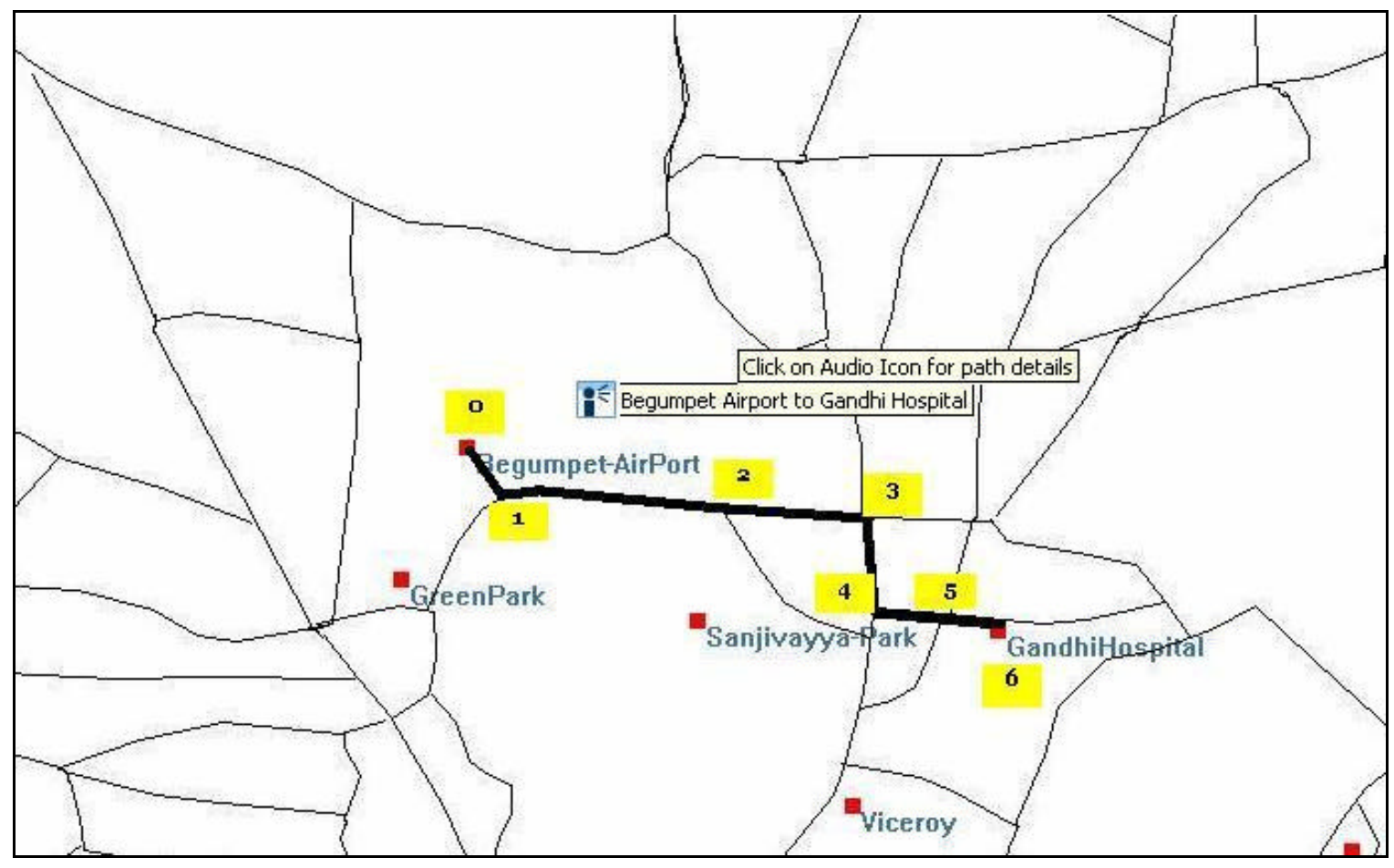

Figure1: MmCIS showing the shortest path between two locations.

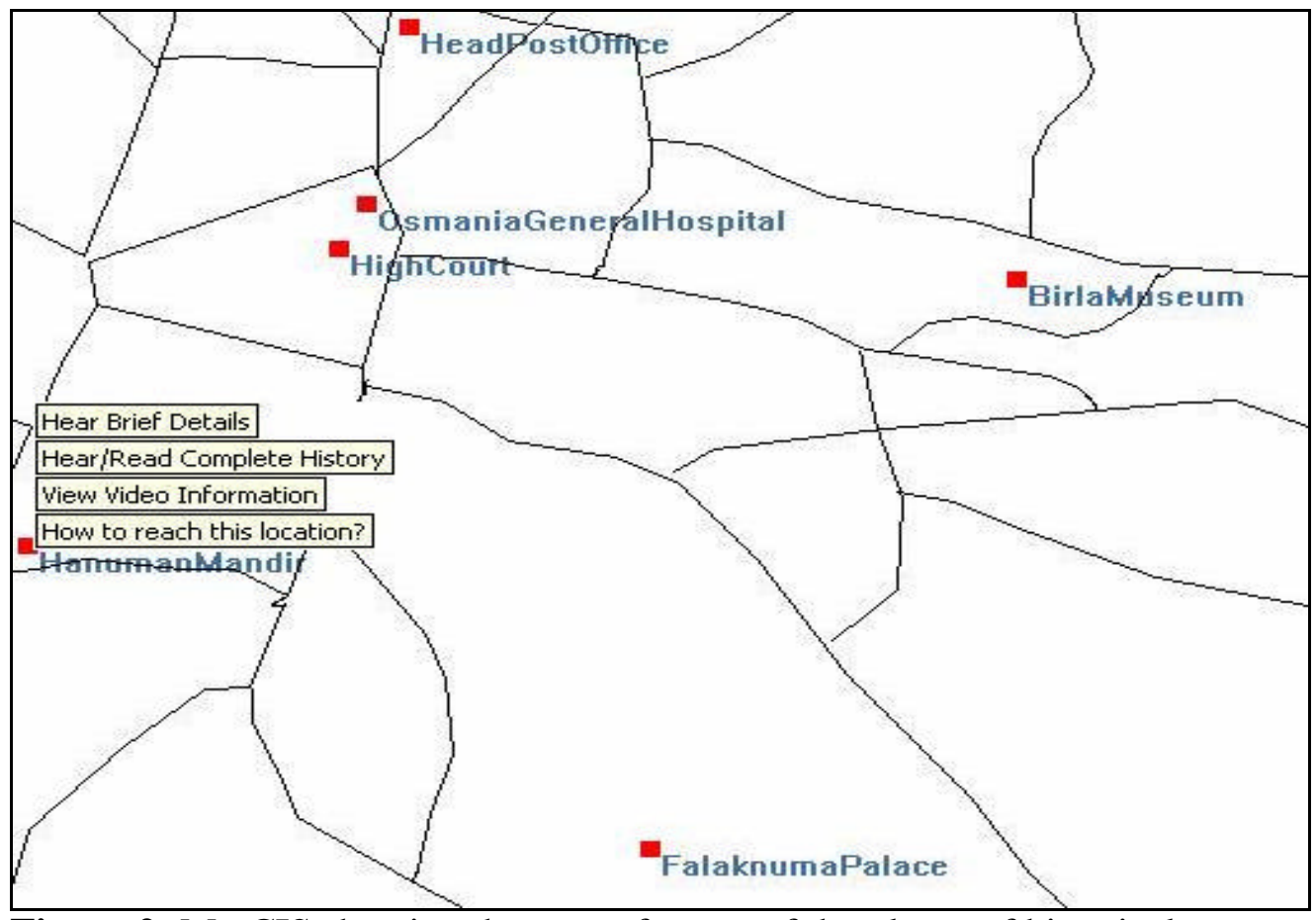

Figure 2: MmCIS showing the menu for one of the places of historical importance. 


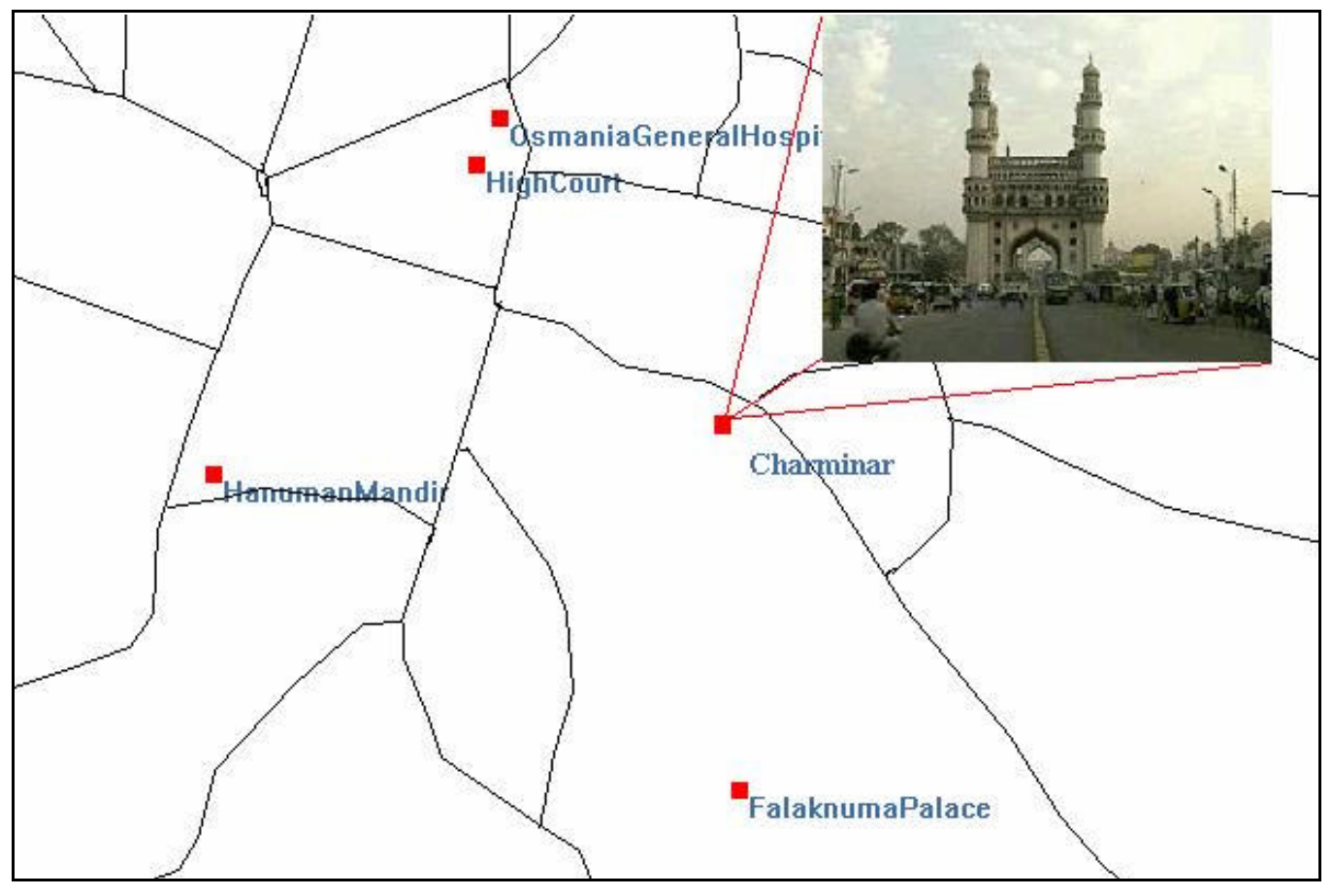

Figure 3: MmCIS showing video information of a historically prominent place. 